\title{
Cancer immunotherapy-associated hypophysitis
}

Franklin Castillero1, Omar Castillo-Fernández*,1, Geiner Jiménez-Jiménez², José Fallas-Ramírez ${ }^{3}$, Marco P Peralta-Álvarez $z^{4,5}$ \& Oscar Arrieta ${ }^{4,5}$

${ }^{1}$ Oncology Department, Instituto Oncológico Nacional de Panamá, 04433, Panamá

${ }^{2}$ Oncology Department, Hospital Dr. Rafael Ángel Calderón Guardia, San José 10101, Costa Rica

${ }^{3}$ Instituto de Investigaciones Farmacéuticas, Facultad de Farmacia, Universidad de Costa Rica, San José 11501, Costa Rica

${ }^{4}$ Thoracic Oncology Unit, Instituto Nacional de Cancerología (INCan), México City 14080, México

${ }^{5}$ Laboratory of Personalized Medicine, Instituto Nacional de Cancerología (INCan), México City 14080, México

*Author for correspondence: oocastillof@gmail.com

The advances in cancer therapy have included the development of drugs that inhibit immune checkpoint ligands. Two types of immune checkpoint inhibitors, both antibodies that target CTLA-4 and PD-1, have been approved for its use in NSCLC and melanoma as first-line or second-line therapy. Sadly, not desirable consequences of immunotherapy are immune-related adverse events. immune-related hypophysitis is the most common endocrine adverse event after thyroid disfunction. The particularity of endocrine immunerelated adverse events is their non-reversibility, with incidence and prevalence destined to increase in the coming years, particularly if this form of therapy is used in the future for earlier stages of cancer. Therefore, hypophysitis represents a challenge for the physician, sometimes occurring without specific symptomatology and which should be considered for clinical management. In this review, we describe the current data regarding the pathophysiology and management for immune-related hypophysitis.

First draft submitted: 21 February 2019; Accepted for publication: 11 July 2019; Published online: 19 August 2019

Keywords: immunotherapy • hypophysitis • immune-related adverse events • PD-1 • CTLA-4

In recent times, novel developed immunotherapeutic drugs have demonstrated a significant potential for the treatment of a wide number of malignancies, including melanoma, lung cancer, kidney cancer, among other malignancies [1]. Immune checkpoint inhibitors (ICIs), which release constraints on immune cells to promote antitumor activity, have produced undeniable clinical results, particularly anti-CTLA- 4 and anti-PD-1 antibodies. Nevertheless, the use of immunotherapy (IT) can produce immune-related adverse events (irAEs). Even though endocrinopathies are not the most frequent adverse effects associated with IT, these can be particularly severe. Therefore patients must be carefully monitored during treatment with immunotherapeutic agents [2].

Hypophysitis is an uncommon condition characterized by a chronic inflammatory infiltrate. Afterward, this infiltration is progressively replaced by fibrotic tissue causing both alterations of the pituitary gland architecture and dysfunction [3,4]. This clinical entity was first described by Goudie and Pinkerton in 1962 [3]. Hypophysitis can be categorized into two types according to its etiology in primary hypophysitis, which has autoimmune pathogenesis without a clear cause. In this type, the inflammatory infiltrate originates from and is limited to pituitary tissue. The second type is secondary hypophysitis, which occurs when the inflammatory disorder is secondary to an adjacent or systemic disease with a clearly identified etiological agent or iatrogenic etiology [3,5]. Regarding histopathology, hypophysitis can be classified as lymphocytic, granulomatous, plasmatic and xanthomatous [6-8]. Likewise, there have also been reports of a necrotizing form [9].

Secondary hypophysitis is associated with cancer immunotherapy, particularly with monoclonal antibodies blocking CTLA-4 and PD-1 molecules [10,11]. Althoug the frequency of endocrine dysfunction is not well defined, hypophysitis correspond to one of most common endocrine irAEs. On the other hand, complications such as thyroiditis, renal insufficiency, diabetes mellitus, colitis, rashes, diabetes insipidus, among other complications, have been reported as well. 


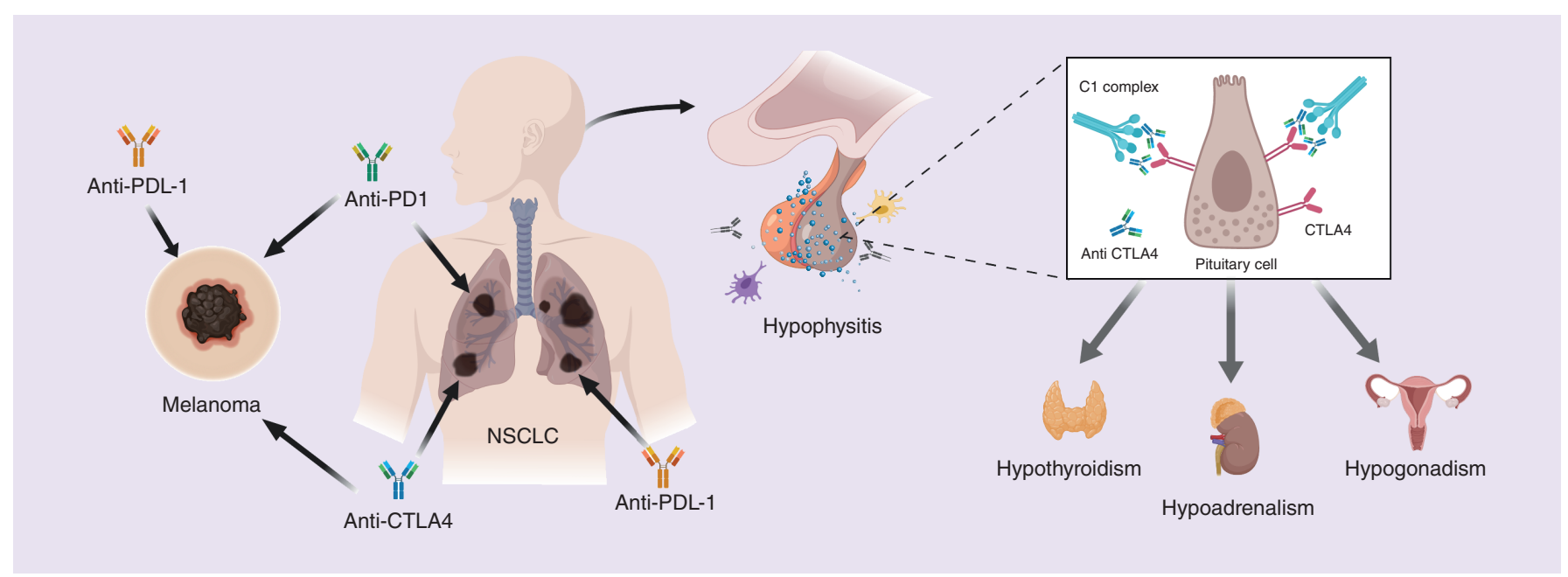

Figure 1. Immune-related hypophysitis pathophysiology. Novel drugs for melanoma and NSCLC treatment (anti-PDL1, anti-CTLA-4, anti-PD1). Pituitary cell ectopic CTLA-4 expression and anti-CTLA-4 induced complement reactivity. Endocrine consequences after IRH: hypogonadism, hypothyroidism, hypoadrenalism.

According to a recent meta-analysis, the frequency of secondary hypophysitis during cancer treatment depends on the type of ICIs used and if they are administered in monotherapy or in combination with other forms of therapy $(0.5-10.5 \%)$ [6]. As the use of ICIs in clinical practice has become more frequent and oncologists are intentionally looking for this clinical entity, immune-related hypophysitis (IRH) incidence has increased [12]. In addition, IRH can induce several endocrine complications, therefore an early recognition, diagnosis, and treatment should be recommended [13]. This review aims to guide the medical oncologist using IT on their clinical practice on the basic mechanisms underlying IRH and to help to suspect this complication in order to make a timely diagnosis and provide appropriate medical treatment.

\section{Epidemiology of immune-related hypophysitis}

IRH is an endocrinology side effect that has been gaining interest in the last decade, with a reported incidence of $0-17 \%$ in all patients exposed to ICIs [14]. Its incidence is more frequent in men, with a M:F ratio of 4:1 [9] and older people, while it is not necessarily dose related [13]. Since 2011, 84 reports and at least 220 well-documented cases of IRH have been reported, most of them regarding the use of Ipilimumab [15]. In fact, the incidence of IRH varies according to the type of ICIs administered .

A meta-analysis by de Filette et al. reported a pooled estimated incidence of IRH of 5.6\% (95\% CI: 3.9-8.1) with the use of ipilimumab versus $1.8 \%$ (95\% CI: 1.1-2.9) for tremelimumab, both anti-CTLA-4 drugs inhibitors [6]. On the other hand, the incidence of IRH with the use of anti-PD-1 drugs nivolumab and pembrolizumab is lower $(0.5 \%$ and 1.1 , respectively). As expected, the highest estimated incidence has occurred with the combination therapy, ranging from $8.8 \%$ (95\% CI: 6.2-12.4) in patients under treatment with nivolumab plus ipilimumab and $10.5 \%$ (95\% CI: 6.5-16.4) in patients receiving pembrolizumab plus ipilimumab [6]. Additionally, at least two cases have been reported with the use of a novel anti-PD-L1, atezolizumab [15]. Moreover, a recent analysis of spontaneous reports submitted to the US FDA Adverse Events Reporting System database reported 191 cases related to ipilimumab, 22 cases for nivolumab, 11 for pembrolizumab and 21 related to the combination of nivolumab + ipilimumab, highlighting the strong relationship of IRH to CTLA-4 inhibitors [16].

\section{Physiopathology of immune-related hypophysitis}

Although the precise pathogenesis of IRH remains unknown and varies according to the mechanism of action of each ICIs [8,13], in vitro and murine model studies have shown a potential involvement of both antibody-dependent cell-mediated cytotoxicity and the complement pathway for IRH develpment [14]. (Figure 1) Iwama et al. were the first to report the ectopic expression of the CTLA-4 antigen on pituitary cells in the hypophysis during IT. Interestingly, anti-CTLA- 4 antibodies can bind to their ectopic ligand in the hypophysis and induce a Type II mediated hypersensitivity reaction. This mechanism leads to continuous classical pathway complement activation, 
which results in tissular damage and explains the chronic evolution of IRH. (Figure 1) In addition, the presence of anti-pituitary antibodies, mainly anti-thyroid stimulating hormone (TSH) and less frequently, directed to Adrenocorticotropic hormone (ACTH) or follicle-stimulating hormone (FSH) have been documented as well [17]. Moreover, Caturegli et al. have also proposed a mechanism based on Type IV hypersensitivity reaction (lymphocytemediated), with the presence of infiltrating lymphocytes and follicle aggregates in pathologic specimens, although more experimental evidence will be required to confirm this findings [9].

\section{Physiopathology associated with anti-CTLA-4 therapy}

Several studies demonstrated ICIs induce the activation of the immune system through their interference capacity against inhibitory activation ligands in different immune regulatory cells (i.e., regulatory T-lymphocytes). Unfortunately this capacity can potentially trigger autoimmune responses against self-antigens as well [17]. CTLA- 4 is a constitutive and broadly expressed protein in different type of immune cells, such as helper (T-CD4 ${ }^{+}$) and cytotoxic $\mathrm{T}$ cells $\left(\mathrm{T}-\mathrm{CD} 8^{+}\right)$. Further, recent studies report CTLA- 4 expression in pituitary adenomas and normal pituitary tissue $[13,18]$. (Figure 1) Traditionally, CTLA-4 has higher affinity for CD80 and CD86 molecules than CD28, which consequently impedes T-cell activation $[19,20]$. Although the use of anti CTLA- 4 antibodies hinder mononuclear cells activation, their use as IT has been associated with the deposition of complement $(\mathrm{C} 1 \mathrm{q})$ in pituitary cells located in the adeno-hypophysis. (Figure 1) Likewise, the presence of serum antibodies against thyrotropic, gonadotropic and corticotropic cells has been reported in patients who develop hypophysitis following the use of ipilimumab [17]. Furthermore, some authors suggest endocrine events (irAEs) caused by these type of antibodies are part of a Type II hypersensitivity reaction. This reaction between cell surface ectopic CTLA- 4 antigen and CTLA- 4 antibodies can activate antibody-dependent cytotoxicity or the classical complement pathway cascade, started by IgG and C1q, and consequently bringing C3, C3d, C4d which leads to other inflammatory cells migration. Interestingly, the induced complement activation can be higher in patients who receive IgG1 type antibodies, such as ipilimumab, versus IgG2 type antibody (tremelimumab) with has a lower complement activation capacity. This could explain the different incidence of hypophysitis among ICIs with the same target [21,22].

\section{Physiopathology associated with anti-PD1/PDL1 therapy}

The incidence of IRH with the use of anti-PD-1/PD-L1 antibodies is considerably lower than the reported with anti-CTLA-4 antibodies [23]. This observed difference could be explained by the fact that there is no ectopic PD-1 or PD-L1 expression in normal hypophysis tissue $[8,18]$. Furthermore, it has been hypothesized PD-1/PD-L1 pathway predominantly regulate inflammatory reactions both in peripheral tissues as well as in the tumour microenvironment. Therefore, the inhibition of this molecular pathway could involve mainly peripheral scenarios [18]. Another difference in these mechanisms is related to the ability of anti-CTLA- 4 to generate new reactive effector T cells against the pituitary gland, which diversifies the autoimmune repertoire; while conversely, anti-PD-1/PD-L1 activate only existing effector cells [2,24]. Although, Mei et al. recently demonstrated the presence of PD-L1 in pituitary adenomas, particularly in functional adenomas, this expression may be due the presence of inflammatory cytokines or different cell-signalling pathways, such as phosphatase and tensin (PTEN), epidermal growth factor receptor (EGFR) or B-Raf proto-oncogene (BRAF) [25]. Suggesting another potential mechanism for the genesis of this complication with the use of PD-1 inhibitors.

\section{New advances}

The expression of CTLA- 4 can vary secondary to genetic polymorphisms, something that has been related to the risk for developing certain autoimmune diseases, such as Graves' disease or Hashimoto's thyroiditis [26], and could explain the variable ectopic expression of CTLA-4 in the pituitary gland. In fact, a Copper-64-based radiotracer was recently developed to evaluate CTLA-4 expression in tissues not previosuly exposed to ICIs, by using positron emission tomography/computed tomography (PET/CT). Hence, if the development of IRH can be predicted in patients with the uptake of this marker in the pituitary gland, this could be useful to avoid any unnecessary exposure to ICIs and consequently irAEs [27]. But currently, this technology is still under development.

\section{Clinical presentation}

Virtually all human organs can present irAEs after ICIs therapy. However, IRH is considered a rare event that is only suspected and diagnosed when severe, such as grade 3 or 4 [2]. The clinical manifestations of IRH usually occur between 6 and 12 weeks after the start of anti-CTLA- 4 therapy [1,17], but can also start later $(8-12$ cycles 
Table 1. Common terminology criteria for immunotherapy side effects applicable for IRH

\begin{tabular}{|c|c|c|c|c|}
\hline Grade 1 & Grade 2 & Grade 3 & Grade 4 & Grade 5 \\
\hline $\begin{array}{l}\text { Asymptomatic or mild } \\
\text { symptoms; clinical or diagnostic } \\
\text { observations only; intervention } \\
\text { not indicated }\end{array}$ & $\begin{array}{l}\text { Moderate; minimal, local or } \\
\text { noninvasive intervention } \\
\text { indicated; limiting age } \\
\text { appropriate instrumental } \\
\text { activities }\end{array}$ & $\begin{array}{l}\text { Severe or medically significant } \\
\text { but not immediately } \\
\text { life-threatening; } \\
\text { hospitalization or prolongation } \\
\text { of existing hospitalization } \\
\text { indicated; limiting self-care }\end{array}$ & $\begin{array}{l}\text { Life-threatening consequences; } \\
\text { urgent intervention indicated }\end{array}$ & Death \\
\hline
\end{tabular}

\begin{tabular}{|c|c|c|c|c|}
\hline $\begin{array}{l}\text { Immune checkpoint inhibitor } \\
\text { type }\end{array}$ & Incidence & Onset & Reported symptoms & Pituitary axis affected \\
\hline Anti-CTLA-4 & $\begin{array}{l}\text { Ipilumumab: } 5.6 \% \text { (95\% Cl: } \\
\text { 3.9-8.1) } \\
\text { Tremelimumab: } 1.8 \%(95 \% \mathrm{Cl}: \\
\text { 1.1-2.9) }\end{array}$ & $6-12$ weeks & $\begin{array}{l}\text { Fatigue, headache, weakness, } \\
\text { vision changes }\end{array}$ & $\begin{array}{l}\text { Hypoadrenalism, hypothyroidism, } \\
\text { hypogonadism }\end{array}$ \\
\hline Anti-CTLA-4 + Anti-PD-1 & $\begin{array}{l}\text { Ipilumumab + nivolumab: } \\
8.8 \% \text { ( } 95 \% \mathrm{Cl} \text { : } 6.2-12.4) \\
\text { Ipilumumab + pembrolizumab: } \\
10.5 \% \text { ( } 95 \% \mathrm{Cl}: 6.5-16.4)\end{array}$ & $10 \pm 5$ weeks & $\begin{array}{l}\text { Headache, blurred vision, } \\
\text { fatigue, hypotension, weakness }\end{array}$ & $\begin{array}{l}\text { Hypoadrenalism, hypogonadism, } \\
\text { hypothyroidism }\end{array}$ \\
\hline Anti - PD-1 & $\begin{array}{l}\text { Nivolumab: } 0.5 \% \\
\text { Pembrolizumab: } 1.1 \%\end{array}$ & 14-36 weeks & $\begin{array}{l}\text { lethargy, fatigue, weight loss, } \\
\text { poor appetite, hypotension, } \\
\text { nausea, vomiting, blurred } \\
\text { vision }\end{array}$ & Hypoadrenalism, hypothyroidism \\
\hline Anti - PD-L1 & Atezolizumab: 2 reported cases & $52-56$ weeks & $\begin{array}{l}\text { Appetite loss, general malaise, } \\
\text { diarrhea }\end{array}$ & Hypoadrenalism \\
\hline
\end{tabular}

of treatment) and insidiously with the use of anti-PD-1 therapy [16]. Generally, symptoms are varied in their presentation and origin and the clinical picture is usually characterized by headache, dizziness, fatigue and/or muscle weakness $[8,28]$, which requires maintaining a high index of suspicion, given the fact that this clinical data could be attributed to the primary disease (Figure 2). Even though, there may be manifestations of endocrine dysfunction, such as fatigue, weight gain/loss, dysthymia, intolerance to cold, constipation and changes in the tone of the voice $[12,29,30]$. Some authors have proposed the exhaustive search for red flag symptoms that indicates the need to speed up timely therapy, as well as the presence of adrenal insufficiency. These red flag symptoms include headaches (described as being predominantly frontal), visual disturbances and arterial hypotension [9]. The latter symptoms may require treatment with vasopressor drugs and, in the context of dehydration and other symptoms of systemic hypoperfusion, could represent an imminent adrenal crisis, which could ultimately endanger the life of patients [31]. Furthermore, other less frequent symptoms have been described, such as constipation, mood changes, arthritis and cognitive impairment which should be taken into account $[32,33]$.

There are some peculiarities regarding clinical presentation according to the type of ICIs involved. (Table 2) In the case of IRH secondary to CTLA-4 blockage, most patients will have headache $(60 \%)$, blurred vision (3\%) and hypocortisolism (91\%), in addition to hypothyroidism (84\%) or hypogonadism $(83 \%)$ [9]. On the other hand, in the IRH associated with PD1 blockage, headache is not usual, and nonspecific symptoms such as fatigue, edema, nausea and blurred vision have been reported. Clinical suspicion in these cases is usually raised by findings of hypothyroidism or hypocortisolism in laboratory tests [34]. Finally, in reported cases related to PD-L1 blockage, the presentation is even later (after 18-19 treatment cycles, or 52-56 weeks), with most nonspecific symptoms (loss of appetite, diarrhea and general discomfort) and apparently with a predilection to affect only the adrenocortical axis [35].

Regarding the severity of irAEs, the National Cancer Institute of the United States has proposed a classification for IRH, like other secondary endocrinopathies caused by the use of IT. This classification goes from grade 1 with asymptomatic or mild symptomatology, to grade 5 which causes death (Table 1) $[24,28]$.

\section{Diagnosis \& clinical approach}

By considering hypophysitis as a potential adverse effect, prevention should focus on the adequate knowledge of ICIs drugs and the incidence of irAEs, providing timely information to the patient, and an exhaustive search for the 


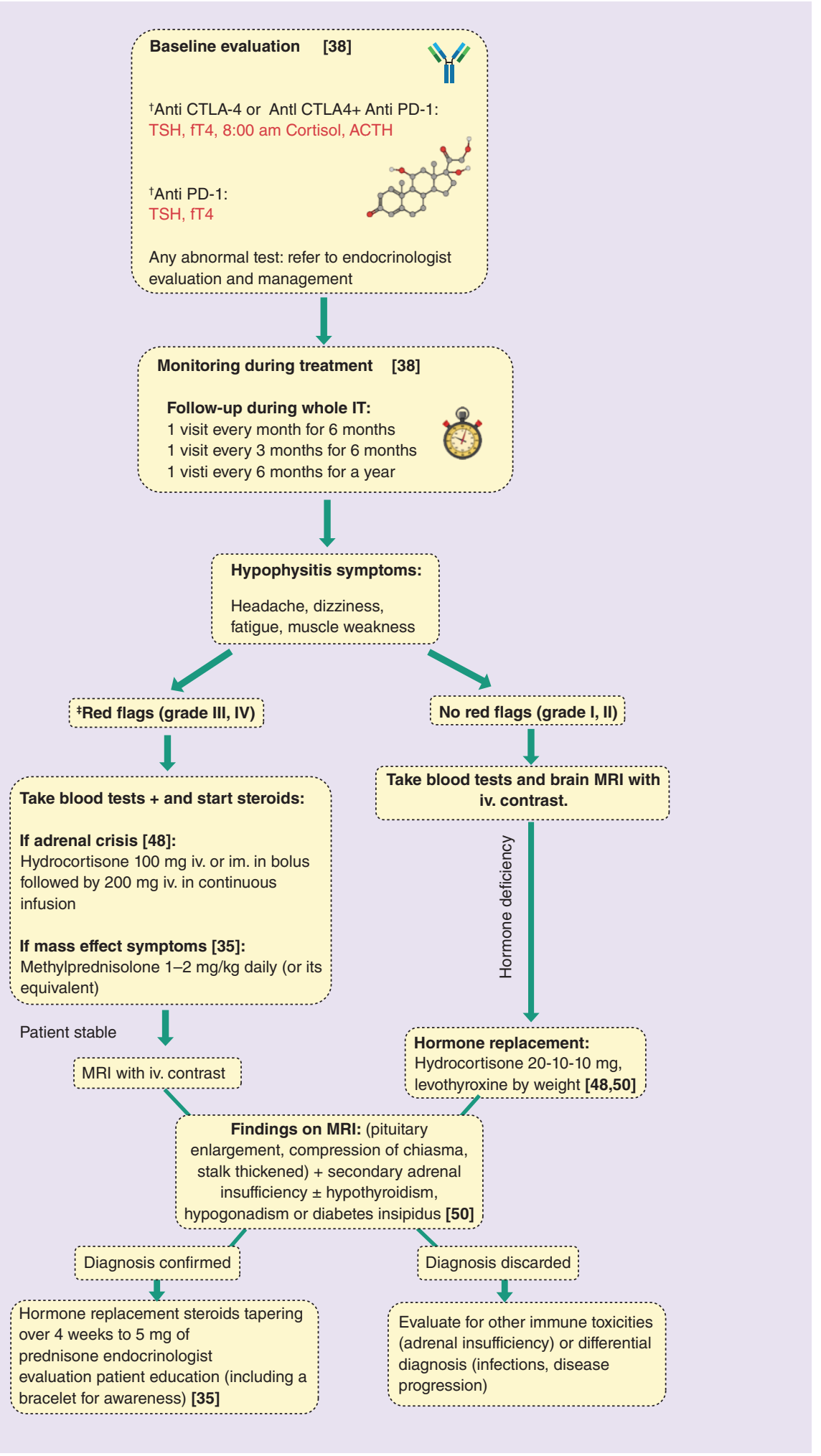

Figure 2. Immune-related hypophysitis diagnosis and treatment. A baseline endocrine evaluation is recommended before the start of IT. In cases of severe symptoms, an immediate use of steroids and intravenous rehydration is encouraged. In a patient with mild symptoms, a diagnostic approach could be made before the start of hormone replacement therapy.

${ }^{\dagger}$ Additional monthly clinical assessment, including symptoms of hypophysitis is encouraged.

${ }^{\ddagger}$ Red flags: frontal headache, new visual symptoms, systolic blood pressure $\leq 90 \mathrm{~mm} \mathrm{Hg}$, severe electrolyte disturbances [35].

Blood test should include: TSH, fT4, ACTH, cortisol, LH, FSH [50]. 
Table 3. Hormone deficiency-related to hypophysitis.

\begin{tabular}{|c|c|c|}
\hline Hormone deficiency & Diagnostic work up & Treatment \\
\hline Hypothyroidism & $\downarrow \mathrm{fT} 4+\downarrow$ or normal TSH & Levothyroxine: $0.5-1.5 \mathrm{mg} / \mathrm{kg}$ \\
\hline Hypoadrenalism & $\begin{array}{l}\downarrow \text { ACTH }+\downarrow \text { serum cortisol }(<250 \mathrm{nmol} / \mathrm{l} \text { if } 9: 00 \mathrm{am}, \\
<150 \mathrm{nmol} / \mathrm{l} \text { if random cortisol }+ \text { symptoms) }\end{array}$ & $\begin{array}{l}\text { Hydrocortisone: } 20,10,10 \mathrm{mg} \text { (must start several days } \\
\text { before thyroid replacement) }\end{array}$ \\
\hline Hypogonadism & $\downarrow$ Testosterone or stradiol $+\downarrow$ LH or FSH & Refer for endocrinologist evaluation ${ }^{\dagger}$ \\
\hline
\end{tabular}

patient's family history for autoimmune diseases [35,36]. The anticipation of this adverse event allows some baseline parameters to be taken into consideration before the start of IT, in order to be able to follow-up and achieve early detection of IRH if presented. Constitutive symptoms should be evaluated as well as basal weight, laboratory tests, including sodium and potassium electrolytes, glycaemia, TSH, free thyroxin (T4), morning cortisol (8:00 am) and ACTH (Figure 2) [37]. These parameters should be evaluated mainly with the use of CTLA-4 inhibitors (particularly ipilimumab). Having in consideration the current evidence, we recommend doing such evaluations on a monthly basis for the first 6 months of treatment, then every 3 months for 6 months and then every 6 months for 1 year; particularly because the monitoring of IRH has been performed in this way in several Phase I studies regarding the approval of ICIs (Figure 2) [38].

The presence of symptoms and clinical suspicion should lead to an expeditious evaluation of complete hormonal function, with special focus in the evaluation of the adrenal and thyroid axes, which are the most commonly affected (Table 3). Generally, in IRH related to CTLA-4 blocking, serum morning cortisol levels are less than $3 \mathrm{mcg} / \mathrm{dl}$ and with ACTH less than $5 \mathrm{pg} / \mathrm{ml}$ [38]. Likewise, the gonadal axis (FSH/luteinizing hormone, estradiol/testosterone) and serum prolactin can also be evaluated [3139]. It is also important to consult the endocrinologist, especially if the patient has been using exogenous steroids chronically, as they may hinder a correct diagnostic approach. Measuring growth hormone and IGF-1 is not routinely recommended.

In clinical practice, hyponatremia (with reported values between $113-134 \mathrm{mEq} / \mathrm{l}$ ) has been reported in up to $56 \%$ of patients receiving IT, which may even contribute to symptoms, but which also corresponds to a finding that should make the diagnosis suspicious in the absence of other clinical data [30]. Other electrolyte disturbances may be present, like hyperkalemia (if adrenal crisis) or hypernatremia (if diabetes insipidus) [38]. The evaluation of visual fields has also been considered useful in patients with visual symptoms. However, in the presence of severe symptoms (grade 2-4), management should be initiated without delay [34,39].

\section{Imaging}

Concerning imaging studies, contrasted magnetic resonance of the pituitary gland is the gold standard to confirm the diagnosis. Radiologists have to pay particular attention to the imaging appearances of this irAE [40]. A diffuse, mild-to-moderate increase in the gland size is usually reported, with homogenous or heterogeneous reinforcement after the administration of contrast. There may also be thickening of the pituitary stalk that may result in a mass effect on the optic chiasm, as well as homogenous contrast enhancement [41]. Additionally, the presence of a 'dural tail' in the contrasted phase adjacent to the gland has been reported [42,43]. A retrospective study with 17 patients with IRH secondary to ipilimumab reported that in $100 \%$ of the subjects, an increase in gland size to a slight or moderate degree was found. Moreover, this finding allowed the clinical diagnosis in 8 patients, weeks before the symptoms were manifested [31]. Afterwards, the pituitary gland diminishes in size between 4 and 12 weeks, producing a subsequent atrophy of the gland. However, it is important to emphasize that a normal MRI does not rule out hypophysitis [8]. PET/CT with fluoro-deoxyglucose has also been useful, showing a focal increase in uptake in the gland and its resolution after treatment with steroids [43].

Although the clinic is fundamental for the suspicion of the underlying diagnosis, it is with the clinical studies and the changes in the MRI that the diagnosis of IRH is reached. Thus, Lam has proposed the presence of one or more of the following manifestations as diagnostic criteria [44]:

- Secondary adrenal insufficiency with acute onset of symptoms;

- Low serum cortisol and low ACTH;

- Secondary hypothyroidism with persistently low free T4 and normal or suppressed TSH;

- Changes in the MRI consistent with hypophysitis. 


\section{Differential diagnosis}

MRI, besides confirming the diagnosis, allows the possibility of ruling out new brain metastases (this being the main differential diagnosis), cerebrovascular events or leptomeningeal disease [3,33,36]. Moreover, it is important to distinguish central pituitary dysfunction from other immune endocrinopathies, such as pituitary macro-adenomas, autoimmune thyroiditis (high TSH, with low T3 and T4), or primary adrenal disease, for example, secondary to metastasis (high or apparently 'normal' ACTH in the scenario of a low serum cortisol) [41,45]. Since central diabetes insipidus, hyperglycemia or diabetic ketoacidosis have been reported in patients with IT, these entities should be discarded [39].

\section{Histology}

Regarding histopathology, there have been several histologic patterns reported in the primary form of hypophysitis (lymphocytic, granulomatous, xanthomatous and plasmacytic, when it is IgG4-related) [7,8]. However, there is a lack of information about the pathologic findings in ICI-related hypophysitis. Caturegli, however, described a necrotizing form of the disease in one case, and lymphocyte infiltrates with follicular aggregates in another [45].

\section{Treatment}

Steroids at high doses have been considered the cornerstone in the management of patients with critical illness (grades 3-4), either related to hypophysitis or hypopituitarism, and with signs and symptoms of significant hyponatremia, severe headache, visual abnormalities or pituitary growth that protrudes or makes mass effect in the optic chiasm [5,36]. In this clinical scenario, once the hypophysitis diagnosis is clear, immunotherapy must be withdrawn, and the indicated management immediately initiated (Figure 2).

In cases of severe mass effect symptoms or severe hypoadrenalism, the use of methylprednisolone at a dose of $1-2 \mathrm{mg} / \mathrm{kg}$ iv. per day for 3-5 days is recommended by the European Society of Medical Oncology guidelines for management of immunotherapy toxicities [36], followed by oral prednisone $1-2 \mathrm{mg} / \mathrm{kg}$, with gradual tapering in 4 weeks [13]. An alternative option is the use of dexamethasone $4 \mathrm{mg}$ iv. every $6 \mathrm{~h}$ for 7 days, followed by gradual titration up to $0.5 \mathrm{mg} /$ day and then making a switch to hydrocortisone at equivalent doses [18]. If the patient presents to the emergency department with an adrenal crisis (i.e., with systolic blood pressure below $90 \mathrm{mmHg}$, hyponatremia, hyperkalemia, tachycardia and postural hypotension), the Society for Endocrinology Endocrine Emergency recommends the use of hydrocortisone in an intravenous bolus of $100 \mathrm{mg}$, followed by a continuous infusion of $200 \mathrm{mg}$ iv. and aggressive rehydration with isotonic saline infusion [46,47]. This approach is practical in the Emergency department, where patient stabilization is the priority, and is consistent with the guidelines for the management of patients with sepsis, which is a differential diagnosis of these patients, and which recommend the use of hydrocortisone [48]. More recently, American Society of Medical Oncologist guidelines recommended high-dose steroids (prednisone or its equivalent, $1-2 \mathrm{mg} / \mathrm{kg}$ ) for grade 3-4 hypophysitis. They also recommend to be aware on doubling doses as stress dosing for illness (like a surgery) and recommends a medical alert bracelet, so that emergency services could be alerted to start stress dose steroids by medical or surgical emergencies $[49,50]$.

Faje reported in a retrospective evidence that high-dose steroids may reduce the overall survival and time to treatment failure (HR: 0.24; $\mathrm{p}=0.002$ and $0.28, \mathrm{p}=0.001$, respectively) in patients with hypophysitis and metastatic melanoma, and also demonstrated that there is no differences in the time for resolution of pituitary enlargement and the clinical symptoms with the use of high-dose steroids therapy [51], so that, the use of high-dose steroids, even in the high-grade disease is controversial.

In patients with stable conditions (grade 1-2), it is not clear if steroids are necessary, the optimal dose to be used or if they represent an optimal management strategy; however, the guidelines proposed by the European Society of Medical Oncology propose the use of oral steroids at the same dose $(1-2 \mathrm{mg} / \mathrm{kg})$ in the case of grade 2 toxicity; whereas in grade 1, only the respective hormone replacement is recommended [36].

After the resolution of symptoms and tapering of the steroids, long-term replacement is needed in most patients [49]. However, It must be considered that the therapeutic objective of steroids is to mitigate the symptoms caused by inflammation and not to recover pituitary function [37,52]. The management must be multidisciplinary because the complications of long-term steroid use could include opportunistic infections such as Pneumocystis jirovecii pneumonia, steroid myopathy and secondary diabetes [44,53]. It is critical to remember that the dose of steroids should gradually decrease to prevent an adverse reaction, as well as the rebound of autoimmune phenomena.

The presence of hypothyroidism requires replacement with thyroid hormone, but given the risk of precipitating an adrenal crisis, the replacement with levothyroxine should be deferred until the hypocortisolism has been treated [44]. 
The same takes place in the presence of hypogonadotropic hypogonadism in men, when the use of testosterone may be useful. Estrogen replacement may also be considered in premenopausal women who have secondary hypogonadism, although management must be guided by the recommendations of endocrinology [36,44,54].

In certain cases, in which the immunotoxicity is refractory or recurrent, certain nonsteroidal immunosuppressants can be used once immunotherapy has been withdrawn. Drugs such as azathioprine, methotrexate and cyclosporin A have been used successfully in cases resistant to corticosteroids [55,56]. However, these alternatives do not have similar evidence, and correspond mainly to the primary form of autoimmune hypophysitis. Its long-term response must be corroborated since relapses have been described after 3 months of use. In the context of chiasma compression, surgical treatment with a trans-sphenoidal approach may be required [56,57].

The use of radiotherapy and radiosurgery to reduce pituitary size has been documented in some cases of primary autoimmune hypophysitis, as well as the use of rituximab, which has been used successfully in cases of primary refractory hypophysitis $[3,58]$. However, there is no clear evidence of its effectiveness in refractory cases of secondary hypophysitis.

One of the concerns that arises in the management of patients who develop hypophysitis is the relevance of withdrawing immunotherapy. Most authors agree that anti-CTLA-4 therapy should be withdrawn in case of symptomatic pan-hypopituitarism and/or grade 3-4 endocrinopathy [23,36,59]. In patients with moderate symptoms (grade 2), the European Association of Clinical Oncology recommends the withdrawal of immunosuppressive treatment and only recommends maintaining it in case of grade 1 symptoms, in which case the anti-CTLA-4 could be continued alongside adequate hormonal replacement therapy (Table 3) [36].

The resumption of ipilimumab after toxicity represents a challenge, and should be considered on an individual basis by achieving a dose of prednisone of less than $7.5 \mathrm{mg} /$ day or its equivalent [44] with strict monitoring and informed consent of the patient [10]. In cases of grade 3 or 4 hypophysitis, some authors recommend discontinuing management permanently. However, González-Rodríguez and colleagues recommend that it should be discussed collaboratively between the treating oncologist and the consulted endocrinologist [18]. The same would apply for inhibitors of PD-L1, although with these, pituitary toxicity is much rarer $[11,58,60,61]$.

Patient monitoring should include affected axes (adrenal, thyroid, gonadal). Adrenal insufficiency associated with hypophysitis secondary to anti-CTLA-4 therapy is usually permanent, so these patients will typically require lifelong management with steroid replacement [13]. The thyroid axis, on the other hand, can recover between 6 and $64 \%$, while the recovery of the gonadal axis is between 11 and $57 \%$. It must be taken into account that the underlying treatment for cancer can also affect these axes, so it can be difficult to differentiate whether gonadal or thyroid deficiency is secondary to hypophysitis or the underlying disease.

\section{Future perspective}

IT with ICIs has become the standard of treatment across a wide variety of oncological indications in the first and second line of treatment for several malignancies. Nowadays, it is available in routine clinical practice for advanced non-small-cell lung cancer (NSCLC), melanoma and many other type of cancers. Sadly, the expertise in the management of irAEs is currently limited to sites that have been involved in the use of ICIs in clinical trials. IRH is a complication that, although rare, represents a main indication for treatment interruption. The frequency of this complication is currently increasing and can lead to severe clinical consequences as endocrine irAEs are commonly

\section{Executive summary}

- The use of immune checkpoint inhibitors for cancer treatment is increasing, and frequently result in endocrine adverse effects, including hypophysitis.

- Oncologists should be ready to detect cases of hypophysitis in a timely manner and provide specific treatment to these patients.

- Hypophysitis should always be considered in immunotherapy-treated patients if unexplained fatigue, headaches, hyponatremia and/or hypotension take place.

- Management should include discontinuation of the immune checkpoint blockade, initiation of corticosteroid therapy for high-grade disease and eventually hormone replacement therapy.

- Hypophysitis impacts treatment of the neoplasm and frequently requires long-term management of this toxic effect.

- A multidisciplinary team approach is essential to insure the accurate diagnosis and management of immune-related hypophysitis. 
non-reversible. Therefore, as the aetiology of this complication is not yet fully understood, its proper diagnosis requires a high index of clinical suspicion, a multidisciplinary approach, imaging assessment and an individualized treatment-based hormone replacement. Hence it is tremendously important to make more research that explains the basic mechanisms underlying this complication and to have individualized clinical guidelines that help clinical decision making on this particular setting.

\section{Author contributions}

O Castillo and F Castillero, conceived the project. All authors were involved in the manuscript design, literature review, manuscript writing, review and final approval.

\section{Financial \& competing interests disclosure}

No funding was received for the preparation of this article. Dr Arrieta reports personal fees and nonfinancial support from Roche, personal fees and nonfinancial support from Lilly, personal fees from Bristol-Myers Squibb, personal fees from Pfizer, grants and personal fees from AstraZeneca, personal fees from Merck, outside the submitted work. The authors have no other relevant affiliations or financial involvement with any organization or entity with a financial interest in or financial conflict with the subject matter or materials discussed in the manuscript apart from those disclosed.

No writing assistance was utilized in the production of this manuscript.

\section{Open Access}

This work is licensed under the Attribution-NonCommercial-NoDerivatives 4.0 Unported License. To view a copy of this license, visit http://creativecommons.org/licenses/by-nc-nd/4.0/.

\section{References}

Papers of special notes have been highlighted as: $\bullet \bullet$ of considerable interest

1. Gedye C, van der Westhuizen A, John T. Checkpoint immunotherapy for cancer: superior survival, unaccustomed toxicities. Intern. Med J. 45(7), 696-701 (2015).

2. Roberts K, Culleton V, Lwin Z, O’Byrne K, Hughes BG. Immune checkpoint inhibitors: navigating a new paradigm of treatment toxicities. Asia Pac. J. Clin. Oncol. 13(4), 277-288 (2017).

3. Guaraldi F, Giordano R, Grottoli S, Ghizzoni L, Arvat E, Ghigo E. Pituitary autoimmunity. In: Endocrine Immunology. Savino W, Guaraldi F (Eds). Karger Publishers, Basel, 48-68 (2017).

4. Glezer A, Paraiba DB, Bronstein MD. Rare sellar lesions. Endocrinol. Metab. Clin. North Am.37, 195-211 (2008).

5. Torino F, Barnabei A, De Vecchis L, Salvatori R, Corsello SM. Hypophysitis induced by monoclonal antibodies to cytotoxic T lymphocyte antigen 4: challenges from a new cause of a rare disease. Oncologist 17(4), 525-535 (2012).

6. de Filette J, Andreescu C, Cools F, Bravenboer B, Velkeniers B. A systematic review and meta-analysis of endocrine-related adverse events associated with immune checkpoint inhibitors. Horm. Metab. Res. 51(03), 145-156 (2019).

7. Chang L-S, Barroso-Sousa R, Tolaney SM, Hodi FS, Kaiser UB, Min L. Endocrine toxicity of cancer immunotherapy targeting immune checkpoints. Endrocr. Rev. 40, 17-65 (2018).

8. Byun DJ, Wolchok JD, Rosenberg LM, Girotra M. Cancer immunotherapy - immune checkpoint blockade and associated endocrinopathies. Nat. Rev. Endocrinol. 13(4), 195-207 (2017).

9. Caturegli P, Di Dalmazi G, Lombardi M et al. Hmiypophysitis secondary to cytotoxic T-lymphocyte-associated protein 4 blockade. Am. J. Pathol. 186(12), 3225-3235 (2016).

10. Illouz F, Briet C, Cloix Let al. Endocrine toxicity of immune checkpoint inhibitors: essential crosstalk between endocrinologists and oncologists. Cancer Med. 6(8), 1923-1929 (2017).

11. O'Kane GM, Labbé C, Doherty MK, Young K, Albaba H, Leighl NB. Monitoring and management of immune-related adverse events associated with programmed cell death protein-1 axis inhibitors in lung cancer. Oncologist 22(1), 70-80 (2017).

12. Solinas C, Porcu M, De Silva Pet al. Cancer immunotherapy-associated hypophysitis. Semin. Oncol. 45(3), 181-186 (2018).

-. Provides meaningful data regarding hypophysitis.

13. Faje A. Immunotherapy and hypophysitis: clinical presentation, treatment, and biologic insights. Pituitary 19, 82-92 (2016).

-• Contains relevant information on the clinical characteristics of hypophysitis.

14. Joshi MN, Whitelaw BC, Palomar MTP, Wu Y, Carroll PV. Immune checkpoint inhibitor-related hypophysitis and endocrine dysfunction: clinical review. Clin. Endocrinol. (Oxf.) 85(3), 331-339 (2016).

-• Contains substantial information about the endocrine dysfunction related with immunotherapy and provides relevant clinical data regarding hypophysitis. 
15. Tan MH, Iyengar R, Mizokami-Stout K. et al. Spectrum of immune checkpoint inhibitors-induced endocrinopathies in cancer patients: a scoping review of case reports. Clin. Diabetes Endocrinol. 5, 1 (2019).

16. Ji H, Tang X, Dong Z, Song L, Jia Y. Adverse event profiles of anti-CTLA-4 and anti-PD-1 monoclonal antibodies alone or in combination: analysis of spontaneous reports submitted to FAERS. Clin. Drug Invest. 39(3), 319-330 (2019).

17. Iwama S, De Remigis A, Callahan MK, Slovin SF, Wolchok JD, Caturegli P. Pituitary expression of CTLA-4 mediates hypophysitis secondary to administration of CTLA-4 blocking antibody. Sci. Transl. Med. 6(230), 230ra45 (2014).

18. González-Rodríguez E, Rodríguez-Abreu D. Spanish group for cancer immuno-biotherapy (GETICA). Immune checkpoint inhibitors: review and management of endocrine adverse events. Oncologist 21(7), 804-816 (2016).

19. Thomas IJ, Petrich de Marquesini LG, Ravanan R et al. CD86 has sustained costimulatory effects on CD8 T cells. J. Immunol. 179(9), 5936-5946 (2007).

20. Contardi E, Palmisano GL, Tazzari PL et al. CTLA-4 is constitutively expressed on tumor cells and can trigger apoptosis upon ligand interaction. Int. J. Cancer 117(4), 538-550 (2005).

21. Quirk SK, Shure AK, Agrawal DK. Immune-mediated adverse events of anticytotoxic T lymphocyte-associated antigen 4 antibody therapy in metastatic melanoma. Transl. Res. 166(5), 412-424 (2015).

22. Bertrand A, Kostine M, Barnetche T, Truchetet M-E, Schaeverbeke T. Immune related adverse events associated with anti-CTLA-4 antibodies: systematic review and meta-analysis. BMC Med. 13, 211 (2015).

23. Corsello SM, Barnabei A, Marchetti P, De Vecchis L, Salvatori R, Torino F. Endocrine side effects induced by immune checkpoint inhibitors. J. Clin. Endocrinol. Metab. 98(4), 1361-1375 (2013).

-. Contains substantial information about the endocrine dysfunction related with immunotherapy.

24. Shang Y hong, Zhang Y, Li J hua, Li P, Zhang X. Risk of endocrine adverse events in cancer patients treated with PD-1 inhibitors: a systematic review and meta-analysis. Immunotherapy 9(3), 261-272 (2017).

25. Mei Y, Bi WL, Greenwald NF et al. Increased expression of programmed death ligand 1 (PD-L1) in human pituitary tumors. Oncotarget 7(47), 76565-7676 (2016).

26. Gough SCL, Walker LSK, Sansom DM. CTLA4 gene polymorphism and autoimmunity. Immunol Rev. 204(1), 102-115 (2005).

27. Higashikawa K, Yagi K, Watanabe K et al. 64Cu-DOTA-anti-CTLA-4 mAb enabled PET visualization of CTLA-4 on the T-cell infiltrating tumor tissues. PLoS ONE 9(11), e109866 (2014).

28. Lomax AJ, McNeil C. Acute management of autoimmune toxicity in cancer patients on immunotherapy: common toxicities and the approach for the emergency physician. Emerg. Med. Australas. 29(2), 245-251 (2017).

29. Andrews S, Holden R. Characteristics and management of immunerelated adverse effects associated with ipilimumab, a new immunotherapy for metastatic melanoma. Cancer Manag. Res. 4, 299-307 (2012).

30. Faje AT, Sullivan R, Lawrence Det al. Ipilimumab-induced hypophysitis: a detailed longitudinal analysis in a large cohort of patients with metastatic melanoma. Journal of Clinical Endocrinology and Metabolism. Oxford University Press, 99, 4078-4085 (2014).

31. Kähler KC, Hassel JC, Heinzerling L et al. Management of side effects of immune checkpoint blockade by anti-CTLA-4 and anti-PD-1 antibodies in metastatic melanoma. J. Dtsch Dermatol. Ges. 14(7), 662-681 (2016).

32. Joshi MN, Whitelaw BC, Palomar MTP, Wu Y, Carroll P V. Immune checkpoint inhibitor-related hypophysitis and endocrine dysfunction: clinical review. Clin. Endocrinol. (Oxf) 85(3), 331-339 (2016).

33. Araujo PB, Coelho M C A, Arruda M, Gadelha M R, Neto L V. Ipilimumab-induced hypophysitis: review of the literature. J. Endocrinol. Invest. 38, 1159-1166 (2015).

34. Lin C-H, Chen K-H, Chen K-Y, Shih S-R, Lu J-Y. Immune checkpoint inhibitor therapy-induced hypophysitis $\sim$ a case series of Taiwanese patients. J. Formos. Med. Assoc. 118(1), 524-529 (2019).

35. Lammert A, Schneider H, Bergmann T et al. Hypophysitis caused by ipilimumab in cancer patients: hormone replacement or immunosuppressive therapy. Exp. Clin. Endocrinol. Diabetes 121(10), 581-587 (2013).

36. Haanen JBAG, Carbonnel F, Robert C et al. Management of toxicities from immunotherapy: ESMO Clinical Practice Guidelines for diagnosis, treatment and follow-up. Ann. Oncol. 28(Suppl. 4), iv119-iv142 (2017).

-. Contains the guidelines for the treatment of adverse events related to immunotherapy.

37. Champiat S, Lambotte O, Barreau E et al. Management of immune checkpoint blockade dysimmune toxicities: a collaborative position paper. Ann. Oncol. 27, 559-574 (2016).

38. Girotra M, Hansen A, Farooki A et al. The current understanding of the endocrine effects from immune checkpoint inhibitors and recommendations for management. JNCI Cancer Spectr. 2(3), 1-9 (2018).

39. Sznol M, Postow MA, Davies MJ et al. Endocrine-related adverse events associated with immune checkpoint blockade and expert insights on their management. Cancer Treat. Rev. 58, 70-76 (2017).

40. Beer L, Hochmair M, Prosch H. Pitfalls in the radiological response assessment of immunotherapy. In: Memo - Magazine of European Medical Oncology. Springer, 138-143 (2018). 
41. Widmann G, Nguyen VA, Plaickner J, Jaschke W. Imaging features of toxicities by immune checkpoint inhibitors in cancer therapy. Curr. Radiol. Rep. 5(11), 59 (2016).

42. Carpenter KJ, Murtagh RD, Lilienfeld H, Weber J, Murtagh FR. Ipilimumab-induced hypophysitis: MR imaging findings. Am. J. Neuroradiol. 30(9), 1751-1753 (2009).

43. Chodakiewitz Y, Brown S, Boxerman JL, Brody JM, Rogg JM. Ipilimumab treatment associated pituitary hypophysitis: clinical presentation and imaging diagnosis. Clin. Neurol. Neurosurg. 125, 125-130 (2014).

44. Lam T, Chan MMK, Sweeting AN et al. Ipilimumab-induced hypophysitis in melanoma patients: an Australian case series. Intern. Med. J. 45(10), 1066-1073 (2015).

45. Caturegli P, Di Dalmazi G, Lombardi M et al. Hypophysitis secondary to cytotoxic T-lymphocyte-associated protein 4 blockade: insights into pathogenesis from an autopsy series. Am. J. Pathol. 186(12), 3225-3235 (2016).

46. Higham CE, Olsson-Brown A, Carroll P et al. Society for endocrinology endocrine emergency guidance: acute management of the endocrine complications of checkpoint inhibitor therapy. Endocr. Connect. 7(7), G1-7 (2018).

47. Annane D, Renault A, Brun-Buisson C et al. Hydrocortisone plus fludrocortisone for adults with septic shock. N. Engl. J. Med. 378(9), 809-818 (2018).

48. Brahmer JR, Lacchetti C, Schneider BJ et al. Management of immune-related adverse events in patients treated with immune checkpoint inhibitor therapy: American Society of Clinical Oncology Clinical Practice Guideline. J. Clin. Oncol. 36(17), 1714-1768 (2018).

49. Brilli L, Danielli R, Ciuoli C et al. Prevalence of hypophysitis in a cohort of patients with metastatic melanoma and prostate cancer treated with ipilimumab. Endocrine 58(3), 535-541 (2017).

50. Faje AT, Lawrence D, Flaherty K et al. High-dose glucocorticoids for the treatment of ipilimumab-induced hypophysitis is associated with reduced survival in patients with melanoma: steroids and hypophysitis outcomes. Cancer 124(18), 3706-3714 (2018).

51. Albarel F, Gaudy C, Castinetti F et al. Long-term follow-up of ipilimumab-induced hypophysitis, a common adverse event of the anti-CTLA-4 antibody in melanoma. Eur. J. Endocrinol. 172(2), 195-204 (2015).

52. Weber JS, Dummer R, de Pril V, Lebbé C, Hodi FS. Patterns of onset and resolution of immune-related adverse events of special interest with ipilimumab. Cancer 119(9), 1675-1682 (2013).

53. Johnson DB, Friedman DL, Berry E et al. Survivorship in immune therapy: assessing chronic immune toxicities, health outcomes, and functional status among long-term ipilimumab survivors at a single referral center. Cancer Immunol. Res. 3(5), 464-469 (2015).

54. Bellastella G, Maiorino MI, Bizzarro Aet al. Revisitation of autoimmune hypophysitis: knowledge and uncertainties on pathophysiological and clinical aspects. Pituitary 19(6), 625-642 (2016).

\section{-• Provides meaningful information about hypophysitis physiopathology.}

55. Curtò L, Torre ML, Cotta OR et al. Lymphocytic hypophysitis: differential diagnosis and effects of high-dose pulse steroids, followed by azathioprine, on the pituitary mass and endocrine abnormalities-report of a case and literature review. ScientificWorldJournal 10, 126-134 (2010).

56. Honegger J, Buchfelder M, Schlaffer S et al. Treatment of primary hypophysitis in Germany. J. Clin. Endocrinol. Metab. 100(9), 3460-3469 (2015).

57. Gu WJ, Zhang Q, Zhu J, Li J, Wei SH, Mu YM. Rituximab was used to treat recurrent IgG4-related hypophysitis with ophthalmopathy as the initial presentation: a case report and literature review. Medicine 96(24), e6934 (2017).

58. Juszczak A, Gupta A, Karavitaki N, Middleton MR, Grossman AB. Ipilimumab: a novel immunomodulating therapy causing autoimmune hypophysitis: a case report and review. Eur. J. Endocrinol. 167(1), 1-5 (2012).

59. Herbst RS, Baas P, Kim DW et al. Pembrolizumab versus docetaxel for previously treated, PD-L1-positive, advanced non-small-cell lung cancer (KEYNOTE-010): a randomised controlled trial. Lancet 387(10027), 1540-1550 (2016).

60. June $\mathrm{CH}$, Warshauer JT, Bluestone JA. Is autoimmunity the Achilles' heel of cancer immunotherapy? Nat. Med. 23(5), 540-547 (2017).

61. NIH, National Cancer Institute. Common Terminology Criteria for Adverse Events

(CTCAE) (2017). https://ctep.cancer.gov/protocoldevelopment/electronic_applications/docs/CTCAE_v5_Quick_Reference_8.5x11.pdf 
